\title{
NGHIÊN CỨU CÁC ĐẶC TRƯNG CƠ BẢN CỦA MẠTT QUASIGEOID
}

\author{
HÀ MINH HOÀ \\ Viện Khoa học Đo đạc và Bản đồ
}

\section{Tóm tắt:}

Bài báo khoa học này đã nghiên cứu việc sử dụng phương trình cơ sở của mặt quasigeoid để giải quyết hàng loạt bài toán như xác định thế trọng trường $W_{0}$ của mặt geoid cục bộ tại trạm nghiệm triều 0 , chuyển đổi mô hình Dị thường độ cao toàn cầu được xác định từ mô hình EGM, mô hình Địa hình động lực trung bình toàn cầu MDT từ mặt geoid toàn cầu về mặt geoid cục bộ phục vụ việc xây dựng các mô hình quasigeoid cục bộ độ chính xác cao, mô hình Địa hình động lực trung bình cục bộ trên vùng biển quốc gia hoặc khu vực. Các kết quả nghiên cứu nêu trên đã được thử nghiệm trong thực tế ở Việt Nam.

\section{1. Đặt vấn đề}

Như chúng ta đã biết, việc giải quyết các bài toán biên hỗn hợp của Trắc địa vật lý đòi hỏi phải thỏa mãn hai điều kiện cơ bản của là:

Điều kiện 1. Các khối lượng vật chất nằm trên mặt biên phải bị loại bỏ;

Điều kiện 2. Các trị đo trên mặt vật lý Trái đất phải được quy chiếu xuống mặt biên và mặt biên luôn là mặt cần xác định.

Vào năm 1849 nhà toán lý người Anh George Gabriel Stokes (1819 - 1903) trong công trình (Stokes G.G.(1849)) đã đề xuất việc giải quyết bài toán biên hỗn hợp với mặt biên là mặt geoid toàn trong trọng trường thực của Trái đất. Bản thân mặt geoid toàn cầu nằm sâu trong lòng đất trên các lục địa, các đảo và các quần đảo. Như vậy dựa trên điều kiện 1 của bài toán biên, để sử dụng mặt geoid toàn cầu làm mặt biên, chúng ta bắt buộc phải loại bỏ các khối lượng vật chất địa hình nằm trên mặt geoid này bao gồm khối lượng vật chất khí quyển nằm trên mặt vật lý Trái đất và khối lượng vật chất địa hình nằm giữa mặt vật lý Trái đất và mặt geoid toàn cầu. Để đáp ứng được điều kiện 2 chúng ta phải biết quy luận phân bố vật chất trong khối lượng vật chất địa hình. Những điều nêu trên là những vấn đề nan giải và tạo nên "hai vấn đề của Stokes G.".

Để khắc phục các hạn chế trong cách tiếp cận của Stokes G., vào năm 1945 trong công trình (Molodenxkii M.X. (1945)), nhà trắc địa vật lý người Nga Mikhil Sergeevich Molodenxkii (1909 - 1991) đã đề xuất giải quyết bài toán biên hỗn hợp trong trọng trường chuẩn của ellipsoid toàn cầu với mặt biên là mặt telluroid mà mỗi điểm $\mathrm{N}$ trên mặt này cách điểm $\mathrm{M}$ tương ứng trên mặt vật lý Trái đất một khoảng cách bằng dị thường độ cao toàn cầu $\bar{\zeta}_{M}$ và cách điểm điểm $Q_{0}$ tương ứng trên mặt ellipsoid toàn cầu một khoảng cách bằng độ cao chuẩn toàn cầu $\bar{H}_{M}^{\gamma}$ (xem hình 1$)$.

Khi sử dụng mặt biên là mặt telluroid nằm khá gần mặt vật lý Trái đất, Molodenxkii M.X. đã làm đơn giản hóa việc thực hiện điều kiện 1 so với cách tiếp cận của Stokes G.. Khi bình luận về cách tiếp cận của Molodenxkii M.X. trong việc sử dụng trọng trường chuẩn

Ngày nhận bài: 05/9/2016, ngày chuyển phản biện: 08/9/2016, ngày chấp nhận phản biện: 16/9/2016, ngày chấp nhận đăng: 19/9/2016 
của ellipsoid toàn cầu để xây dựng mặt telluroid và giải quyết bài toán biên hỗn hợp, trong các tài liệu (jekeli C. (2000; Matt, A., 2010)) đã cho đánh giá rằng tính đến khả năng xác định độ cao với điều kiện tránh được giả thuyết về phân bố mật độ vật chất trong lớp vỏ Trái đất cần sử dụng trọng trường xấp xỉ với trọng trường Trái đất. Trọng trường chuẩn của ellipsoid thích hợp với điều kiện này khi ellipsoid toàn cầu chứa toàn bộ khối lượng của Trái đất (bao gồm cả khối lượng vật chất khí quyển), quay quanh bán trục nhỏ với tốc độ quay trung bình của Trái đất và mặt ellipsoid này là mặt đẳng thế của trọng trường chuẩn. Thực tế, độ cao chuẩn toàn cầu và dị thường độ cao toàn cầu liên hệ với mặt telluroid và được xác định trong trọng trường chuẩn của ellipsoid toàn cầu (xem các tài liệu Pellinen L.P. (1978), Ogorodova L.V. (2010), trg. 66; Ahmed A. E.M. (2013)).

Công thức kinh điển của lý thuyết độ cao trong trọng trường thực của Trái đất có dạng:

$$
\bar{W}_{0}-W_{M}=\int_{o}^{M} g \cdot d h,
$$

ở đây $\bar{W}_{0}$ - thế trọng trường thực tại điểm $O$ trên mặt geoid toàn cầu; $W_{M}$ - thế trọng trường thực tại điểm $M$ trên mặt vật lý Trái đất; $\mathrm{g}$ và dh là gia tốc lực trọng trường và chênh cao được đo trên mỗi đoạn độ cao của đường thủy chuẩn OM.

Chúng ta ký hiệu $U_{0}$ là thế trọng trường chuẩn trên mặt của ellipsoid toàn cầu. Giả thiết rằng chênh cao đo dh trên mỗi đoạn đo trong trọng trường thực của Trái đất đã được chuyển thành chênh cao $d h^{\gamma}$ trong trọng trường chuẩn của ellipsoid sao cho $\bar{\gamma} \cdot d h^{\gamma}=g$. $d h$ (Pellinen L.P. (1978)). Khi đó đối với điểm $\mathrm{N}$ nằm trên mặt telluroid và có thế trọng trường chuẩn $U_{N}$, hiệu các giá trị thế trọng trường chuẩn $U_{0}-U_{N}$ được xác định theo công thức (Simberov B.P. (1975)):

$$
U_{0}-U_{N}=\int_{Q_{0}}^{N} \gamma \cdot d h^{\gamma}=\bar{\gamma}_{M} \cdot \bar{H}_{M}^{\gamma},
$$

ở đây ${ }^{\gamma}$ và $d h^{\gamma}$ là gia tốc lực trọng trường chuẩn và chênh cao của đoạn đo trong trọng trường chuẩn của ellipsoid Trái đất trung bình, $U_{0}$ - thế trọng trường chuẩn trên mặt ellipsoid, $\bar{H}_{M}^{\gamma}$ - độ cao chuẩn toàn cầu của điểm $\mathrm{M}, \bar{\gamma}_{M}$ - giá trị trung bình của gia tốc lực trọng trường chuẩn trên đoạn $Q_{0} N$

Để thiết lập mối quan hệ giữa độ cao chuẩn $\bar{H}_{M}^{\gamma}$ trong trọng trường chuẩn của ellipsoid toàn cầu với các trị đo trên mặt vật lý Trái đất, từ (2) lưu ý (1) Molodenxkii M.X đã đưa ra

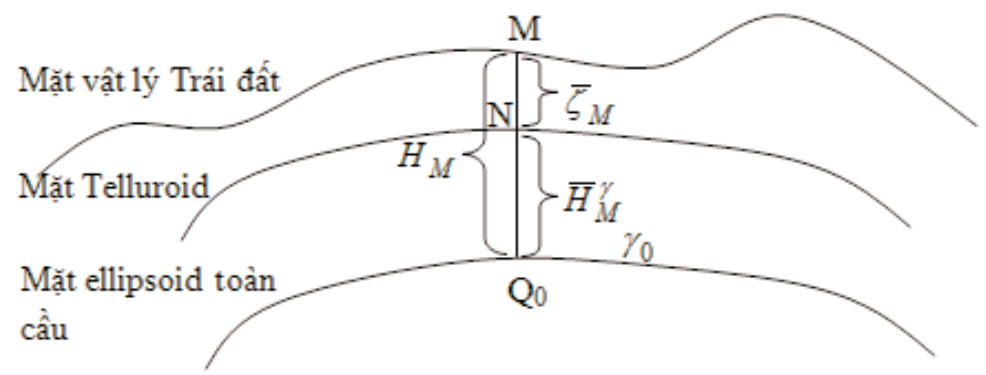

Hình 1: Dị thường độ cao toàn cầu $\bar{\zeta}_{M}$ và độ cao chuẩn toàn cầu $\bar{H}_{M}^{\gamma}$ của điểm $M$ 
điều kiện đối đối với telluroid:

$$
C_{M}=\bar{W}_{0}-W_{M}=U_{0}-U_{N},
$$

ở đây đại lượng $C_{M}$ được gọi là số lượng địa thế (geopotential number).

Sự tồn tại của điều kiện Molodenxkii M.X. (3), theo các tài liệu Simberov B.P. (1975) và Pellinen L.P. (1978), là do sự không trùng nhau giữa trọng trường thực của Trái đất và trọng trường chuẩn của ellipsoid toàn cầu.

Từ điều kiện (3) chúng ta có công thức tính toán độ cao chuẩn:

$$
\bar{H}_{M}^{\gamma}=\frac{C_{M}}{\bar{\gamma}_{M}}=\frac{\bar{W}_{0}-W_{M}}{\bar{\gamma}_{M}}=\frac{U_{0}-U_{N}}{\bar{\gamma}_{M}},
$$

ở đây giá trị trung bình $\bar{\gamma}_{M}$ của gia tốc lực trọng trường chuẩn trên đoạn $Q_{0} N$ được xác định theo công thức:

$$
\bar{\gamma}_{M}=\gamma_{0}-0,1543 \cdot \bar{H}_{M}^{\gamma}+0,036 \cdot 10^{-6} \cdot\left(\bar{H}_{M}^{\gamma}\right)^{2}<m G a l>,
$$

ở đây $\gamma_{0}$ là giá trị gia tốc lực trọng trường chuẩn tại điểm $Q_{0}$ trên mặt ellipsoid toàn cầu.

Lưu ý điều kiện (3), dị thường độ cao toàn cầu $\bar{\zeta}_{M}$ bằng đoạn $\mathrm{MN}$ trên hình 1 trong trọng trường chuẩn của ellipsoid toàn cầu được xác định theo công thức:

$$
\bar{\zeta}_{M}=\frac{U_{N}-U_{M}}{\bar{\gamma}_{M}}=\frac{W_{M}-U_{M}}{\bar{\gamma}_{M}}+\frac{U_{0}-\bar{W}_{0}}{\bar{\gamma}_{M}}=\frac{T_{M}}{\bar{\gamma}_{M}}+\frac{U_{0}-\bar{W}_{0}}{\bar{\gamma}_{M}},
$$

ở đây $U_{M}$ - thế trọng trường chuẩn của điểm $\mathrm{M}$ trên mặt vật lý Trái đất, thế nhiễu tại điểm $\mathrm{M}$ có dạng:

$$
T_{M}=W_{M}-U_{M}
$$

Công thức (6) còn được gọi là công thức Bruns tổng quát. Như vậy lý thuyết độ cao chuẩn được trình bày ở trên hoàn toàn đúng với nhận xét của Yurkina $\mathrm{M}$. I. trong tài liệu (Yurkina M. (1996)) rằng lý thuyết Molodenxkii M.X. được xây dựng trong trường hợp tổng quát khi $U_{0} \neq \bar{W}_{0}$. Nếu ellipsoid toàn cầu là ellipsoid Trái đất trung bình, thì chúng ta có điều kiện $U_{0}=\bar{W}_{0}$. Khi đó từ (6) chúng ta có công thức Bruns $\bar{\zeta}_{M}=T_{M}$ do nhà thiên văn và toán học người Đức Henrich Bruns (1848 - 1919) đề xuất vào năm 1878 trong công trình (Bruns H., 1878).

Điểm qua lý thuyết của Molodenxkii M.X. chúng ta thấy rằng việc sử dụng mặttelluroid làm mặt biên cho phép giải quyết hiệu quả bài toán biên hỗn hợp của Trắc địa vật lý và xây dựng chặt chẽ lý thuyết độ cao chuẩn. Theo tài liệu (Simberov B.P. (1975)), nghiệm của bài toán biên hỗn hợp theo cách tiếp cận của Molodenxkii M.X. là thế nhiễu trên mặt telluroid có giá trị rất gần với thế nhiễu $T_{M}(7)$ trên mặt vật lý Trái đất và thực tế coi chúng bằng nhau. Điều này giải thích lý do của sự phổ biến của lý thuyết của Molodenxkii M.X. trong việc xây dựng các mô hình trọng trường Trái đất EGM hiện nay trên thế giới. Mặc dù lý 
thuyết độ cao chuẩn được xây dựng dựa trên mặt telluroid rất chặt chẽ về mặt lý thuyết, nhưng lý thuyết này không được xây dựng trên thực tế do mặt telluroid không có quan hệ trực tiếp với mặt geoid là mặt đẳng thế trong trọng trường thực của Trái đất và được nhận làm mặt khởi tính cho hệ độ cao theo đề xuất của nhà toán học người Đức Johann Benedict Listing (1808 - 1882) vào năm 1873 trong công trình (Listing J.B. (1873)). Vì lý do này, Molodenxkii M.X. đã đề xuất sử dụng mặt quasigeoid làm mặt khởi tính cho hệ độ cao, thêm vào đó mặt quasigeoid trùng với mặt geoid trên các biển và các đại dương thế giới và càng tách rời khỏi mặt geoid khi càng đi sâu vào đất liền. Trên hình 2 , từ điểm $\mathrm{M}$ trên mặt vật lý Trái đất dựng đường $\mathrm{MQ}_{0}$ cắt mặt quasigeoid toàn cầu tại điểm $\mathrm{S}$. Khi đó điểm $\mathrm{S}$ nằm trên mặt quasigeoid toàn cầu và đoạn $\mathrm{MS}$ bằng độ cao chuẩn $\bar{H}_{M}^{\gamma}(4)$, đoạn $\mathrm{SQ}_{0}$ được gọi là độ cao quasigeoid và có giá trị bằng dị thường độ cao $\bar{\zeta}_{M}(6)$. Tuy nhiên đề xuất của Molodenxkii M.X. mới chỉ xác định mặt quasigeoid về mặt hình học. Như vậy hiện nay chúng ta đang ở trong trạng thái: Khi mô tả lý thuyết độ cao chuẩn chúng ta sử dụng mặt telluroid, khi triển khai hệ độ cao chuẩn trong thực tế chúng ta sử dụng mặt quasigeoid.

Ngày nay, với việc phát triển mạnh mẽ mô hình trọng trường Trái đất EGM, mô hình Địa hình động lực trung bình MDT cùng với công nghệ GNSS, chúng ta đang có công việc liên quan đến cả mặt quasigeoid cục bộ lẫn mặt quasigeoid toàn cầu. Trong lý thuyết của Molodenxkii M.X. việc sử dụng mặt biên là mặt telluroid nhằm giải quyết cả bài toán biên hỗn hợp lẫn xây dựng lý thuyết hệ độ cao chuẩn. Mô hình quasigeoid được sử dụng để giải quyết bài toán biên hỗn hợp do nó nằm sâu trong lớp vỏ Trái đất và việc sử dụng nó làm mặt biên sẽ dẫn đến hai vấn đề của Stokes G. Việc nghiên cứu mô hình toán học của mặt quasigeoid nhằm giải quyết các bài toán xây dựng các mối quan hệ toán học giữa mặt quasigeoid và mặt geoid và giữa mặt quasigeoid cục bộ và mặt quasigeoid toàn cầu. Các mối quan hệ nêu trên đang trở nên cấp thiết trong việc khai thác hiệu quả các mô hình toàn cầu và công nghệ GNSS để giải quyết các bài toán trắc địa vật lý hiện đại ở tầm quốc gia và khu vực. Điều nêu trên cũng là vấn đề khoa học được giải quyết trong bài báo khoa học này.

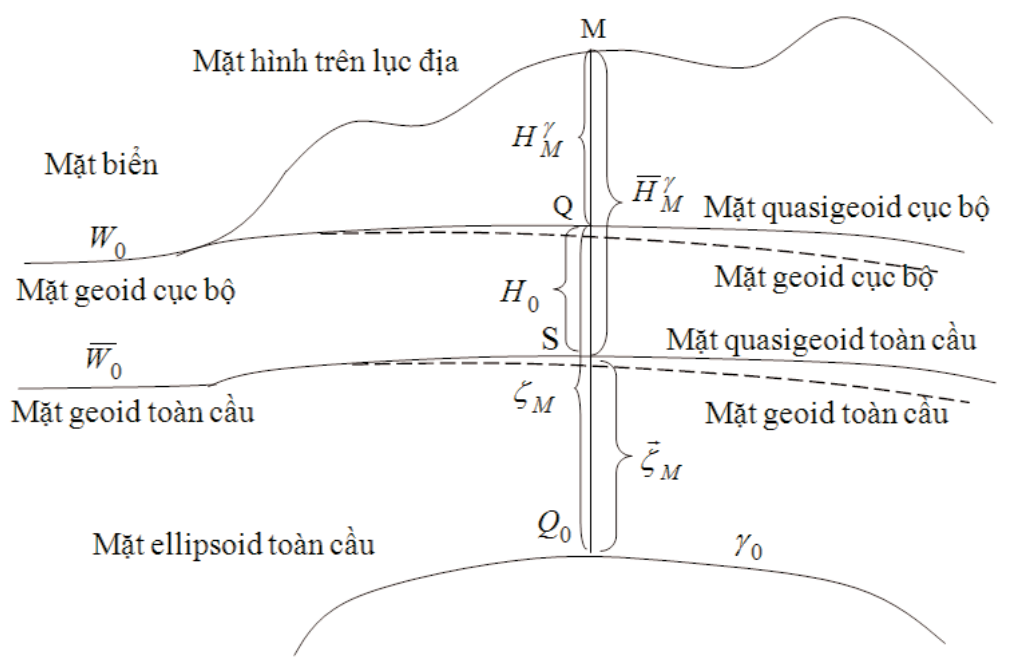

Hình 2: Quan hệ giữa mặt vật lý Trái đất, mặt quasigeoid cục bộ, mặt quasigeoid toàn cầu và mặt ellipsoid toàn cầu 


\section{Giải quyết vấn đề}

Đối với điểm $S$ nằm trên mặt quasigeoid toàn cầu trên hình 2 , trong tài liệu (Hà Minh Hòa. (2010)) đã chứng minh được thế trọng trường chuẩn $U_{S}$ của điểm này ở dạng sau đây:

$$
U_{S}=\bar{W}_{0}-T_{M},
$$

ở đây $\bar{W}_{0}$ - thế trọng trường thực của mặt geoid toàn cầu, $T_{M}$ - thế nhiễu tại điểm $\mathrm{M}$ tương ứng trên mặt vật lý Trái đất.

Công thức (8) được gọi là phương trình cơ sở của mặt quasigeoid. Đối với tập hợp các điểm $M$ trên mặt vật lý Trái đất, các giá trị thế nhiễu $T_{M}(7)$ của chúng không bằng nhau. Do đó từ (8) có thể thấy rằng các giá trị thế trọng trường chuẩn của tập hợp các điểm $\mathrm{S}$ không bằng nhau, tức mặt quasigeoid không phải là mặt đẳng thế trong trọng trường chuẩn của ellipsoid.

Từ công thức (8) lưu ý (3), (7) chúng ta suy ra được điều kiện đối với quasigeoid ở dạng sau:

$$
C_{M}=\bar{W}_{0}-W_{M}=U_{S}-U_{M} .
$$

Khi ký hiệu $\bar{\gamma}_{S M}$ là giá trị trung bình của gia tốc lực trọng trường chuẩn trên đoạn SM trên hình 2 , về nguyên tắc dựa trên quan hệ (9), độ cao chuẩn toàn cầu $\bar{H}_{M}^{\gamma}$ được xác định theo công thức:

$$
\bar{H}_{M}^{\gamma}=\frac{C_{M}}{\bar{\gamma}_{S M}}=\frac{\bar{W}_{0}-W_{M}}{\bar{\gamma}_{S M}}=\frac{U_{S}-U_{M}}{\bar{\gamma}_{S M}} .
$$

Vậy nẩy sinh câu hỏi: giá trị $\bar{\gamma}_{S M}$ có bằng giá trị $\bar{\gamma}_{M}(5)$ hay không ?. Chúng ta sẽ làm rõ vấn đề này. Các giá trị gia tốc lực trọng trường chuẩn tại các điểm $\mathrm{M}$ và $\mathrm{S}$ có dạng sau:

$$
\begin{gathered}
\gamma_{M}=\gamma_{0}-0,3086 \cdot \bar{H}_{M}+0,0723 \cdot 10^{-6} \cdot \bar{H}_{M}^{2} \\
\gamma_{S}=\gamma_{0}-0,3086 \cdot \bar{\zeta}_{M}+0,0723 \cdot 10^{-6} \cdot \bar{\zeta}_{M}^{2} .
\end{gathered}
$$

Giá trị trung bình của hai giá trị nêu trên có dạng sau:

$$
\begin{aligned}
& \bar{\gamma}_{S M}=\frac{\gamma_{M}+\gamma_{S}}{2}=\gamma_{0}-0,1543 \cdot \bar{H}_{M}^{\gamma}+0,03615 \cdot 10^{-6} \cdot\left(\bar{H}_{M}^{\gamma}\right)^{2}-0,3086 \cdot \bar{\zeta}_{M}+ \\
& +0,0723 \cdot 10^{-6} \cdot \bar{H}_{M}^{\gamma} \cdot \bar{\zeta}_{M}+0,0723 \cdot 10^{-6} \bar{\zeta}_{M}^{2} .
\end{aligned}
$$

Khi lấy hiệu $\bar{\gamma}_{M}(5)$ và $\bar{\gamma}_{S M}$

$$
\bar{\gamma}_{M}-\bar{\gamma}_{S M}=0,3086 . \bar{\zeta}_{M}-0,0723 \cdot 10^{-6} \cdot \bar{H}_{M}^{\gamma} \cdot \bar{\zeta}_{M}-0,0723 \cdot 10^{-6} \cdot \bar{\zeta}_{M}^{2} .
$$

và chia hai vế cho tích $\bar{\gamma}_{M} \cdot \bar{\gamma}_{S M}$ chúng ta nhận được biểu thức: 


$$
\frac{1}{\bar{\gamma}_{S M}}-\frac{1}{\bar{\gamma}_{M}}=\frac{0,3086 \cdot \bar{\zeta}_{M}}{\bar{\gamma}_{M} \cdot \bar{\gamma}_{S M}}-\frac{0,0723 \cdot 10^{-6} \cdot \bar{H}_{M}^{\gamma} \cdot \bar{\zeta}_{M}}{\bar{\gamma}_{M} \cdot \bar{\gamma}_{S M}}-\frac{0,0723 \cdot 10^{-6} \cdot \bar{\zeta}_{M}^{2}}{\bar{\gamma}_{M} \cdot \bar{\gamma}_{S M}} .
$$

Cho $\bar{\gamma}_{M}=\bar{\gamma}_{S M}=979764,5 \mathrm{mGal}$ (giá trị trung bình của gia tốc lực trọng trường trên toàn cầu) $\bar{\zeta}_{M}=100 \mathrm{~m}$ và độ cao chuẩn ở đỉnh Everest thuộc dãy Himalaya $\bar{H}_{M}^{\gamma}=8848 \mathrm{~m}$, các thành phần

$$
\frac{0,3086 \cdot \bar{\zeta}_{M}}{\bar{\gamma}_{M} \cdot \bar{\gamma}_{S M}}=3,21 \cdot 10^{-11}, \frac{0,0723 \cdot 10^{-6} \cdot \bar{H}_{M}^{\gamma} \cdot \bar{\zeta}_{M}}{\bar{\gamma}_{M} \cdot \bar{\gamma}_{S M}}=6,66 \cdot 10^{-14},
$$

$\frac{0,0723.10^{-6} \cdot \bar{\zeta}_{M}^{2}}{\bar{\gamma}_{M} \cdot \bar{\gamma}_{S M}}=7,53 \cdot 10^{-16}$ đều là các đại lượng nhỏ bỏ qua. Do đó chúng ta luôn có quan hệ $\frac{1}{\bar{\gamma}_{S M}}=\frac{1}{\bar{\gamma}_{M}}$. Do đó các độ cao chuẩn toàn cầu $\bar{H}_{M}^{\gamma}(4)$ và $\bar{H}_{M}^{\gamma}$ (10) là tương đương nhau, tức

$$
\bar{H}_{M}^{\gamma}=\frac{C_{M}}{\bar{\gamma}_{M}}=\frac{\bar{W}_{0}-W_{M}}{\bar{\gamma}_{M}}=\frac{U_{S}-U_{M}}{\bar{\gamma}_{M}} .
$$

Độ cao quasigeoid toàn cầu trong trọng trường chuẩn của ellipsoid toàn cầu được xác định theo công thức

$$
\bar{\zeta}_{M}=\frac{U_{0}-U_{S}}{\bar{\gamma}_{M}}
$$

Thay $U_{S}(8)$ vào công thức này, chúng ta lại nhận được công thức (6).

Các chứng minh ở trên cho thấy rằng khi sử dụng mặt quasigeoid, việc tính độ cao chuẩn $\bar{H}_{M}^{\gamma}$ theo công thức (4) và dị thường độ cao (độ cao quasigeoid) theo công thức (6) hoàn toàn đúng đắn.

Khi tính đến mối liện hệ giữa mặt quasigeoid và mặt geoid, trong tài liệu (Hà Minh Hòa (2014)) đã sử dụng công thức (9) để nghiên cứu xác định các công thức chuyển độ cao chuẩn, dị thường độ cao giữa các hệ triều.

Chúng ta nghiên cứu một ứng dụng khác của công thức (8). Trên hình 2 đường $M Q_{0}$ cắt mặt quasigeoid cục bộ tại điểm $Q$, thêm vào đó mặt quasigeoid cục bộ này liên hệ với mặt geoid cục bộ có thế trọng trường thực $W_{0}$ tại trạm nghiệm triều 0 . Đoạn $\mathrm{MQ}$ bằng độ cao chuẩn cục bộ $H_{M}^{\gamma}$, còn đoạn $\mathrm{QQ}_{0}$ bằng dị thường độ cao cục bộ $\zeta_{M}$. Tương tự như chứng minh công thức (8), thế trọng trường chuẩn $U_{Q}$ của điểm $Q$ được xác định theo công thức:

Từ (8) và (13) suy ra quan hệ:

$$
U_{Q}=W_{0}-T_{M}
$$

$$
\bar{W}_{0}-W_{0}=U_{S}-U_{Q}
$$


Từ (14) chúng ta nhận được công thức xác định đoạn QS bằng độ cao $\mathrm{H}_{0}$ của mặt quasigeoid cục bộ so với mặt quasigeoid toàn cầu tương ứng với điểm $\mathrm{M}$ ở dạng sau:

$$
H_{0}=\frac{\bar{W}_{0}-W_{0}}{\bar{\gamma}_{M}}=\frac{U_{S}-U_{Q}}{\bar{\gamma}_{M}} \text {. }
$$

Trong công thức (15), đại lượng $\frac{\bar{W}_{0}-W_{0}}{\bar{\gamma}_{M}}$ chính là độ cao $\mathrm{H}_{0}$ của mặt geoid cục bộ so với mặt geoid toàn cầu tại trạm nghiệm triều 0 . Thành phần là độ cao của mặt quasigeoid cục bộ so với mặt quasigeoid toàn cầu tương ứng với điểm $\mathrm{M}$. Chúng ta biết rằng các mặt đẳng thế chuẩn trong trọng trường chuẩn của ellipsoid không song song với nhau. Tuy nhiên mặt quasigeoid trong trọng trường chuẩn của ellipsoid không phải là mặt đẳng thế. Trong ví dụ mặt geoid cục bộ Hòn Dấu ở Việt Nam, các kết quả nghiên cứu lý thuyết và thực nghiệm trong các tài liệu (Hà Minh Hòa và nnk (2012; Ha Minh Hoa (2013); Hà Minh Hòa (2014); Hà Minh Hòa (2016)) cho thấy rằng độ cao $\mathrm{H}_{0}$ của mặt quasigeoid cục bộ Hòn Dấu so với mặt quasigeoid toàn cầu là không đổi không chỉ trên lãnh thổ Việt Nam, mà còn trên toàn cầu.

Chúng ta tiếp tục nghiên cứu ứng dụng khác của các công thức (8) và (13). Đối với giá trị trung bình $\bar{\gamma}_{Q M}$, chứng minh tương tự như đối với giá trị trung bình $\bar{\gamma}_{S M}$ ở trên, chúng ta có quan hệ $\frac{1}{\bar{\gamma}_{Q M}}=\frac{1}{\bar{\gamma}_{M}}$. Do đó độ cao chuẩn cục bộ $H_{M}^{\gamma}$ được xác định theo công thức:

$$
H_{M}^{\gamma}=\frac{W_{0}-W_{M}}{\bar{\gamma}_{M}}=\frac{U_{Q}-U_{M}}{\bar{\gamma}_{M}} .
$$

Từ (11) và (16) lưu ý (14) chúng ta nhận được quan hệ giữa độ cao chuẩn toàn cầu $\bar{H}_{M}^{\gamma}$ và độ cao chuẩn cục bộ $H_{M}^{\gamma}$ ở dạng sau:

$$
\bar{H}_{M}^{\gamma}=\frac{U_{S}-U_{M}}{\bar{\gamma}_{M}}=\frac{U_{Q}-U_{M}}{\bar{\gamma}_{M}}+\frac{\bar{W}_{0}-W_{0}}{\bar{\gamma}_{M}}=H_{M}^{\gamma}+\frac{\bar{W}_{0}-W_{0}}{\bar{\gamma}_{M}} .
$$

Tương tự, dị thường độ cao cục bộ $\zeta_{M}$ được xác định theo công thức:

$$
\zeta_{M}=\frac{U_{0}-U_{Q}}{\bar{\gamma}_{M}}
$$

Từ (12) và (18) lưu ý (14) chúng ta nhận được quan hệ giữa dị thường độ cao cục bộ $\zeta_{M}$ và dị thường độ cao toàn cầu $\bar{\zeta}_{M}$ ở dạng sau:

$$
\zeta_{M}=\frac{U_{0}-U_{Q}}{\bar{\gamma}_{M}}=\frac{U_{0}-U_{S}}{\bar{\gamma}_{M}}+\frac{\bar{W}_{0}-W_{0}}{\bar{\gamma}_{M}}=\bar{\zeta}_{M}+\frac{\bar{W}_{0}-W_{0}}{\bar{\gamma}_{M}} .
$$


Các công thức (17), (19) đã được chứng minh bằng phương pháp khác trong tài liệu (Hà Minh Hòa (2007)) và đã được sử dụng rộng rãi trong các công trình (Hà Minh Hòa và nnk (2012); Hà Minh Hòa (2013); Hà Minh Hòa, Nguyễn Bá Thủy, Phan Trọng Trịnh, Nguyễn Nguyên Cương, Nguyễn Phi Sơn, Nguyễn Thị Thanh Hương và nnk (2016)) để xác định thế trọng trường $W_{0}$ của mặt geoid cục bộ Hòn Dấu dựa trên mặt geoid toàn cầu với thế trọng trường $\bar{W}_{0}=62636856,0 \mathrm{~m}^{2} \mathrm{~s}^{-2}$ và lưu ý sự không đổi của độ cao độ cao $\mathrm{H}_{0}$ của mặt quasigeoid cục bộ Hòn Dấu so với mặt quasigeoid toàn cầu để xây dựng mô hình Mặt biển trung bình MDTVN dựa trên mô hình Địa hình động lực DTU10MDT và mô hình quasigeoid khởi đầu VIGAC2014 dựa trên mô hình geoid toàn cầu EGM2008 ở Việt Nam.

\section{Kết luận}

Việc thiết lập được (8) đã cho phép khai thác được các sản phẩm khoa học - công nghệ hiện đại như mô hình trọng trường Trái đất, mô hình Địa hình động lực trung bình MDT và công nghệ GNSS trong việc giải quyết nhiều bài toán trắc địa vật lý ở tầm quốc gia hoặc khu vực như xây dựng mô hình quasigeoid cục bộ độ chính xác cao, xây dựng mô hình mặt biển trung bình cục bộ MDT trên vùng biển quốc gia nhằm thống nhất hệ độ cao cả trên đất liền và trên vùng biển, xác định thế trọng trường $W_{0}$ của mặt geoid cục bộ để hoàn thiện hệ độ cao hiện đại của quốc gia hoặc khu vực. Các vấn đề được nghiên cứu trong bài báo này đã được triển khai thực nghiệm trong thực tiễn ở Việt Nam. O

\section{Tài liệu tham khảo}

[1]. Ahmed A. E.M. (2013). Normal (Gravimetric) Heights Versus Orthometric Heights. International Journal of Advanced Research In Engineering And Applied Sciences, Vol. 2, No. 11, ISSN: 2278-6252, pp. 68-78.

[2]. Bruns H., 1878. Die Figur der Erde; ein Beitrag zur Europischen Gradmessung. Publikation des Kniglichen Preussischen Geodtischen Instituts, Berlin, p.26.

[3]. Hà Minh Hòa (2007). Giải quyết một số vấn đề liên quan đến việc chuyển hệ độ cao được xác định từ mặt nước biển trung bình ở trạm thủy triều về mặt Quasigeoid toàn cầu. Tạp chí Địa chính số 2 , tháng 4/2007, trg. 3 - 11.

[4]. Hà Minh Hòa. (2010). Tiếp cận khái niệm về mặt Quasigeoid. Tạp chí Khoa học Đo đạc và Bản đồ, No3, 3/2010, trg. 3 - 15.

[5]. Hà Minh Hòa và nnk (2012). Nghiên cứu cơ sở khoa học của việc hoàn thiện hệ độ cao gắn liền với việc xây dựng hệ tọa độ động lực quốc gia. Đề tài khoa học và công nghệ cấp Bộ Tài nguyên và Môi trường giai đoạn 2010 - 2012. Hà Nội - 2012.

[6]. Ha Minh Hoa (2013). Estimating the geopotential value $W_{0}$ of the local geoid based on data from local and global normal heights of GPS/Leveling points in Vietnam. Geodesy and Cartography. Taylor \& Francis. UDK 528.21, doi:10.3846/20296991.2013.823705, V.39 (3): 99-105.

[7]. Hà Minh Hòa (2014). Lý thuyết và thực tiễn của Trọng lực trắc địa. Nhà Xuất bản Khoa học và Kỹ thuật, 592 trg., Hà Nội - 2014.

[8]. Hà Minh Hòa, Nguyễn Bá Thủy, Phan Trọng Trịnh, Nguyễn Nguyên Cương, Nguyễn Phi Sơn, Nguyễn Thị Thanh Hương và nnk (2016). "Nghiên cứu đánh giá các mặt chuẩn 
mực nước biển (mặt "0" độ sâu, trung bình và cao nhất) theo các phương pháp trắc địa, hải văn và kiến tạo hiện đại phục vụ xây dựng các công trình và quy hoạch đới bờ Việt Nam trong xu thế biến đổi khí hậu". Đề tài khoa học và phát triển công nghệ mã số KC.09.19/11 -15 giai đoạn 2012 - 2015 thuộc Chương trình KH\&CN trọng điểm cấp Nhà nước KC-09/11-15 "Nghiên cứu khoa học và công nghệ phục vụ quản lý biển, hải đảo và phát triển kinh tế biến" giai đoạn 2011 - 2015. Bộ Khoa học và Công nghệ, Hà Nội - 2016.

[9]. Hà Minh Hòa (2016). Nghiên cứu sự thay đổi độ cao giữa mặt quasigeoid cục bộ Hòn Dấu và mặt quasigeoid toàn cầu trên phạm vi toàn cầu. Tạp chí Khoa học Đo đạc và Bản đồ, số 28, tháng 06/2016, trg. 1 - 7.

[10]. Hofmann-Wellenhof B. and Moritz H. (2006). Physical Geodesy, 2nd edition, Springer, Wien - New York, ISBN 13: 978-3211335444, 403 p..

[11]. jekeli C. (2000). Heights, the geopotential and vertical datums. Report No. 459. The Ohio State University, Columbus, $35 \mathrm{p}$.

Hofmann-Wellenhof B. and Moritz H. (2006). Physical Geodesy, 2nd edition, Springer, Wien - New York.

[12]. Listing J.B. (1873). Ueber unsere jetzige Kenntuts der Gestalt und der Erde. Nachrichten von der Georg - Augusts Universi 3 : 33-98.

[13]. Matt, A., 2010. New Zealand Vertical Datum 2009. The New Zealand Surveyor, No. 300, pp. 5-16.

[14]. Молоденский М. С. (1945). Основные вопросы геодезической гравиметрии. Труды ЦННИГАиК, № 42, 107 с.

Molodenxkii M.X. (1945). Các vấn đề cơ bản của trọng lực trắc địa. Truđư TXNHIIGAiK, No42, 107 trg. (Tiếng Nga).

[15]. Огородова Л. В., Шимберев Б. П., Юзефрович А. П. (1978). Гравиметрия, М. Недра, 325 c.

Ogorodova L.P., Simberov B.P., luzephovich A.P. (1978). Trọng lực. Matxcơva, "Nedra", 325 trg. (Tiếng Nga).

[16]. Огородова Л. В. (2010). Нормальное поле и определение аномального потенциала. Москва, МИИГАиК, 105 с., ISBN 978-5-91188-025-5.

Ogorodova L.V. (2010). Trường chuẩn và xác định thế dị thường. Matxcơva, MIIGAiK, 105 trg., ISBN 978-5-91188-025-5.

[17]. Пеллинен Л. П. (1978). Высшая геодезия (теоретичесрая геодезия). Москва. Недра, 264 с.

Pellinen L.P. (1978). Trắc địa cao cấp (trắc địa lý thuyết). Matxcơva, Nedra, 264 trg. (Tiếng Nga).

[18]. Шимберов Б. П. (1975). Теория Фигуры Земли. М., Недра, 432 С. Nga).

Simberov B.P. (1975). Lý thuyết Hình dạng Quả đất. Matxcơva, Nedra, 325 trg. (Tiếng (Xem tiếp trang 58) 